\title{
Role of phasiRNAs from two distinct phasing frames of GhMYB2 loci in cis- gene regulation in the cotton genome
}

Ting Zhao ${ }^{1 \dagger}$, Xiaoyuan Tao ${ }^{1 \dagger}$, Menglin Li ${ }^{2}$, Mengtao Gao ${ }^{2}$, Jiedan Chen ${ }^{1}$, Na Zhou ${ }^{2}$, Gaofu Mei ${ }^{2}$, Lei Fang ${ }^{1}$, Linyun Ding ${ }^{2}$, Baoliang Zhou', Tianzhen Zhang ${ }^{1,2}$ and Xueying Guan ${ }^{1,2^{*}}$ (D)

\begin{abstract}
Background: Phased small interfering RNA (phasiRNA) is primarily derived from the 22-nt miRNA targeting loci. GhMYB2, a gene with potential roles in cotton fiber cell fate determination, is a target gene of miR828 and miR858 in the generation of phasiRNAs.

Results: In the presented work, through the evaluation of phasing scores and phasiRNA distribution pattern, we found that phasiRNAs from GhMYB2 were derived from the 3' cleavage fragments of 22-nt miR828 and 21-nt miR858 respectively. These two miRNA targeting sites initiated two phasing frames on transcripts of one locus. By means of RNA ligase-mediated rapid amplification of CDNA ends (RLM-RACE), we further demonstrated that phasiRNAs derived from the two phasing frames played a role in cis-regulation of GhMYB2. The phasiRNAs derived from GhMYB2 were expressed in the somatic tissues, especially in anther and hypocotyl. We further employed our previous small RNA sequencing data as well as the degradome data of cotton fiber bearing ovules, anthers, hypocotyls and embryogenic calli tissues published in public databases, to validate the expression, phasing pattern and functions of phasiRNAs.
\end{abstract}

Conclusions: The presenting research provide insights of the molecular mechanism of phasiRNAs in regulation of GhMYB2 loci.

Keywords: Cotton, Fiber, phasiRNAs, RLM-RACE

\section{Background}

The phased small interfering RNAs (phasiRNAs) exist in a wide variety of plant genomes, from algae and moss species to monocot and dicot species [1]. The first reported phasiRNA was trans-acting small interfering RNA (tasiRNA) derived from long non-coding RNA loci, namely TAS genes: TAS1/2, TAS3 and TAS4 [2]. As a

\footnotetext{
* Correspondence: xueyingguan@zju.edu.cn

${ }^{\dagger}$ Ting Zhao and Xiaoyuan Tao contributed equally to this work.

${ }^{1}$ College of Agriculture and Biotechnology, Zhejiang University, Hangzhou, Zhejiang, China

${ }^{2}$ State Key Laboratory of Crop Genetics and Germplasm Enhancement, Cotton Hybrid R \& D Engineering Center (the Ministry of Education), College of Agriculture, Nanjing Agricultural University, Nanjing, Jiangsu, China
}

specialized type of phasiRNAs, tasiRNAs originate from TAS gene transcripts and functionally act in trans to regulate mRNA at the post-transcriptional level [3]. The miRNAs that initiate phasiRNA generation are known as phasiRNA triggers [4]. TAS1/2 is triggered by miR173 $[2,5,6]$. TAS3 is triggered by miR390 [5, 7-9] and TAS4 by miR828 [10-13]. In addition to triggering by a single miRNA, there is also an alternative pattern of phasiRNA generation, known as the two-hit model [7] and phasiRNA production can be triggered by two distinct small RNAs, as phasiRNA production can be triggered by two distinct small RNA targeting, as observed in the petunia genome [14].

(c) The Author(s). 2020 Open Access This article is licensed under a Creative Commons Attribution 4.0 International License, which permits use, sharing, adaptation, distribution and reproduction in any medium or format, as long as you give appropriate credit to the original author(s) and the source, provide a link to the Creative Commons licence, and indicate if changes were made. The images or other third party material in this article are included in the article's Creative Commons licence, unless indicated otherwise in a credit line to the material. If material is not included in the article's Creative Commons licence and your intended use is not permitted by statutory regulation or exceeds the permitted use, you will need to obtain permission directly from the copyright holder. To view a copy of this licence, visit http://creativecommons.org/licenses/by/4.0/ The Creative Commons Public Domain Dedication waiver (http://creativecommons.org/publicdomain/zero/1.0/) applies to the data made available in this article, unless otherwise stated in a credit line to the data. 
Although the biogenesis of phasiRNAs has been explored in the past decade, our understanding of the expression and phasing pattern of phasiRNAs remains limited. The phasiRNA distribution studies have demonstrated their tissue specific expression pattern. For example, phasiRNAs is the predominant type of small RNA found in the anther of angiosperms [15]. Furthermore, analysis of the small RNA profiles in maize anther shows that phasiRNA expression varies during the cell development [16]. According to the small RNA length, phasiRNA pattern can be divided into 21-nt and 24-nt phasing intervals. 21-nt phasiRNAs are active prior to 24-nt phasiRNAs at various stages of the cell fate specification and differentiation while meiosis is associated with 24-nt phasiRNAs activation. The latest DNA modification assays show that CG, CHG and $\mathrm{CHH}$ methylation levels are high in the phasiRNAs loci for both 21-nt and 24-nt groups in meiocytes [15]. These features suggest that phasiRNAs play an important role in anther development and potentially in epigenetic modification as well.

Recent studies on phasiRNAs in plant suggest that phasiRNAs function as small interfering RNA (siRNA) in critical agronomic trait controlling. For example, miR2118 is expressed in the panicle development stages in rice [17] and other seed plant species [18]. Further forward genetics and transgenic evidences demonstrate that miR2118 triggered Pms1 locus to generate phasiRNAs in determining photoperiod-sensitive male sterility (PSMS) in rice [19]. Selective studies have shown that miR828 targets are involved in abiotic stress responses in Arabidopsis [13], apple [20], and sweet potato [21], as well as in flavonoid biogenesis [22, 23] and epidermal fiber cell development in cotton [24].

The functional pattern of phasiRNA include the cleaving of the target gene sequence according to the reverse complementary manner. Some TAS2-derived siRNAs can recognize pentatricopeptide repeat (PPR) protein coding genes in this way [25]. A recent functional study reports that the rice AGO5 homolog, MEL1, preferentially binds to phasiRNAs [26]. This phenomenon was highly associated with the miR2118-targeted sequences and miR2118-triggered phasiRNAs.

PhasiRNAs in cotton genome remains largely unknown. Eleven genes in Upland cotton were predicted to be TAS genes [27]. Moreover, some 200 phasing loci have been identified in sea-island cotton [28]. Previously, we made the first report of a functional phasiRNAderiving gene in the cotton genome, GhMYB2, targeted by miR828 and miR858 [24]. GhMYB2 encodes a MYB transcriptional factor that plays a role in plant epidermal cell fate determination. Promoter activity assay and mRNA in situ data demonstrate that GhMYB2 expression predominantly occurs in cotton seed fiber cells during the cell differentiation stage [29, 30]. The impact of miR858 on the phasiRNA biogenesis derived from GhMYB2 remains unknown. The function of the GhMYB2-generated phasiRNAs are not clear, yet. Here we examined both small RNA sequencing data from our previous studies of cotton leaf and ovule tissue, as well as mRNA degradome data on cotton fiber-bearing ovules, anthers, hypocotyls and embryogenic calli (EC), which we obtained from an online published database. Reviewing these sources in conjunction, we have further explored the new phasing pattern driven by two microRNA-targeting sites on GhMYB2 and the cis-regulatory role of GhMYB2-derived phasiRNAs during the early stage of cotton fiber differentiation.

\section{Results}

\section{Cotton GhMYB2 generates phasiRNAs in two phases}

PhasiRNA-deriving genes undergo a phase-change after the formation of dsRNA to generate siRNA in-phase, as reported in the model plant, Arabidopsis [31]. To examine whether GhMYB2 can derive siRNA in-phase, we adapted the calculation of phase score to evaluate the level of phasiRNAs of GhMYB2 using the small RNA library of ovule tissue form upland cotton (Gossypium hirsutum, accession Texas Marker-1, TM-1) harvested on 0 DPA (day post anthesis). As shown in Fig. 1a, the phasing curve patterns of GhMYB2 presented clear peaks in phase of $3^{\prime}$ end by miR828 cleavage site. This confirmed our previous reports on GhMYB2 derived phasiRNAs triggered by miR828 [24]. Among the phasiRNAderiving gene candidates, Gh_D07G1901 (miR390 targeting) was predicted to be equivalent to GhphasiRNA1 in the cotton genome. The phasing curve on GhMYB2 was not as regular as those of GhphasiRNA1. Although the 21-nt phasiRNA interval was clearly observed, alternative peaks were also present in ovules and other tissues. Only the action of GhphasiRNA1 in hypocotyls generated a smooth phasing curve at the 21-nt interval (Fig. 1a). We examined the pattern of the alternative peaks by analyzing their intervals. The 21-nt intervals were observed in the curve (Fig. 1a). Further examination of phasiRNA abundance and location indicated that the peaks of the GhMYB2 phasing curve were derived from the 3' cleavage site of the miRNA858 target (Fig. 1b, Supplemental table 1). Moreover, minor peaks were also observed repeatedly in the ovule and anther tissues. Therefore, we confirmed that GhMYB2 can generate siRNA in phase on the $3^{\prime}$ cleavage fragment of both miR828 and miR858. Correspondingly, we named the phasing frame from the 3' cleavage fragment of miR828 Phasing frame 1 (PF1). The alternative phasing frame from miR858 was named Phasing frame 2 (PF2). More phasiRNAs were found in phase of PF2 than PF1, as shown in Fig. 1a and b. 
a

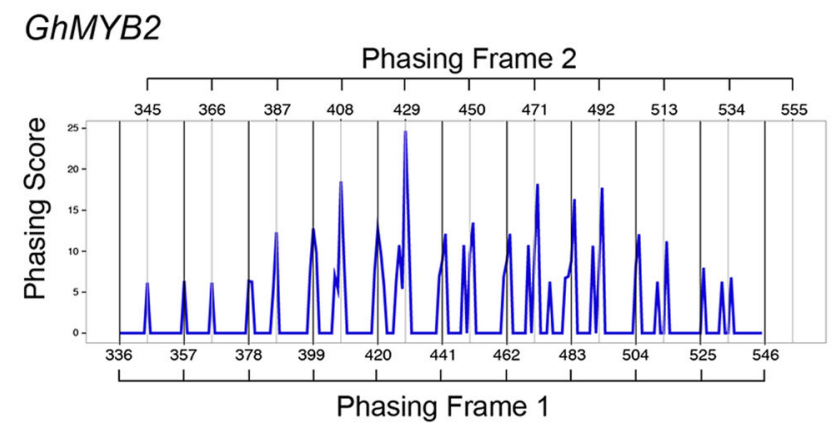

GhphasiRNA1

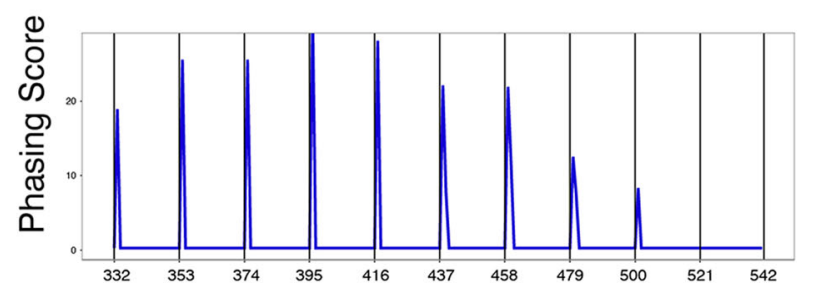

b

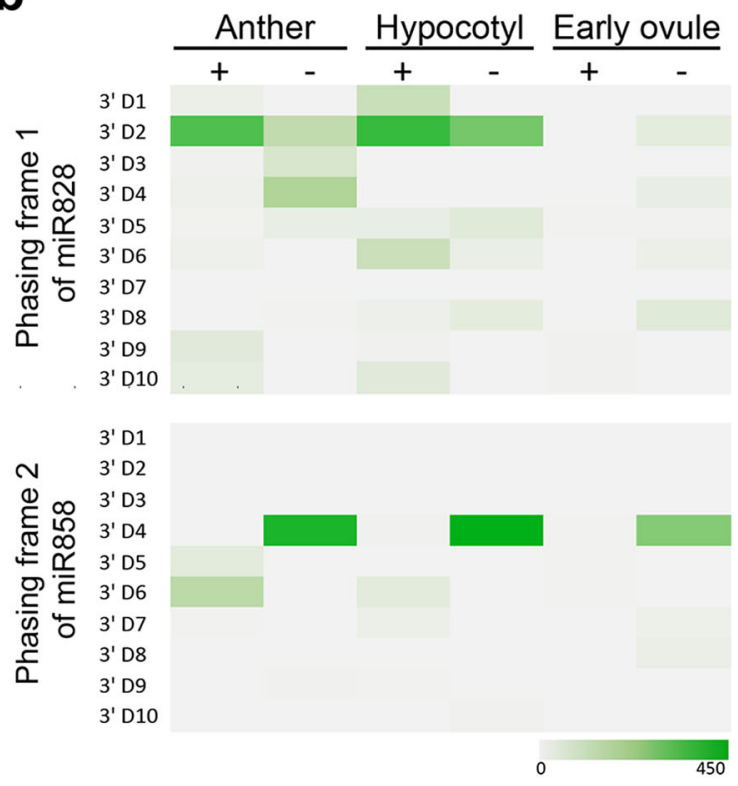

Fig. 1 Alternative phasing of phasiRNAs on the GhMYB2 loci in the cotton genome. a, Phasing score curve (see method) showing Phasing frame 1 (PF1) and Phasing Frame 2 (PF2) at the 21-nt interval on the GhMYB2 loci. FR1 confirmed the phasing changes starting from miR828 cleavage site. $\mathbf{b}$, Heat map showing the phasiRNA density over the 2 phasing frames in anther, hypocotyl and early ovule tissues. The sense and antisense strands are indicated by "+" and "-", respectively

In contrast to the alternative phasing in the two-hit model triggered by miR390 [7], the distance between miR828 and miR858 on GhMYB2 was as short as $12 \mathrm{bp}$. There was insufficient phasing space (21 nt for one small RNA in phase) between these two miRNA targeting sites, given that most of the phasiRNA triggers are 22-nt miRNAs. Due to the observation of phasiRNAs from two phasing frames, the phasing process should initiate from the miR858 and miR828 cleavage fragments respectively on independent transcripts. Given that miR858 itself cannot trigger phasiRNA genesis, the phasing process initiated on miR858 cleavage site on endogenous GhMYB2 might be affected by miR828 triggered phasing change. The two miRNA sites triggered the phasiRNA genesis from two miRNA 3' cleavage fragments should be on distinct starting site.

\section{GhMYB2 -derived phasiRNA can degrade the GhMYB2 by cis- effect}

The two phasing frames of GhMYB2 were visualized as the two major phasing peaks in the phasing score curve (Fig. 2a). These phasiRNAs might accelerate mRNAs degradation by forming dsRNA on the origin loci in cis. To examine this hypothesis, we detected the internal cleavage sites of GhMYB2 mRNA by phasiRNAs. RNA ligase-mediated rapid amplification of cDNA ends (RLM-RACE) was performed using total RNA extracted from anthers in which the phasiRNAs were proactively expressed. Results indicated that
GhMYB2 was cleaved by phasiRNAs generated by both miR828 and miR858. The cleavage sites detected was exactly correspond to the phasiRNA predicted cleavage sites. Cleavage site a (indicated in Fig. 2b) was generated by the PF1-derived phasiRNA, PF1_3' D3(-). The cleavage site was between 10th and 11th nucleotide of PF1_3' D3(-). This pattern was similar with the miRNA-mediated mRNA slicing in plant genome. Similar pattern was observed on cleavage site b, which was generated by the PF2-derived phasiRNA, PF2_3' D8(-). PF2_3' D8(-) also generated another cleavage site $c$ between the 15th and 16th nucleotide. Cleavage site d was on PF2-derived phasiRNA, PF2_3' D10(-). Although this method is not for quantitative determination for cleavage products due to different efficiency in DNA fragment cloning, this results provided a direct evidence that GhMYB2-derived phasiRNAs can degrade the GhMYB2 transcript in cis.

We further detected the degraded fragments of GhMYB2 mRNA in anthers, hypocotyls and embryogenic calli (EC) (Supplemental table 2, seen in Methods). The number of GhMYB2 mapped reads in the anther degradome was dramatically higher than in the hypocotyl and EC degradomes (Supplemental Fig. 2) (Wilcoxon rank sum tests, typically $P<0.01$ ). MYB109, and GhphasiRNA1 did not follow this pattern (Supplemental Fig. 2). These data indicate that GhMYB2 transcripts were preferentially degrades the by phasiRNAs of its own in cis in anthers. 


\section{a

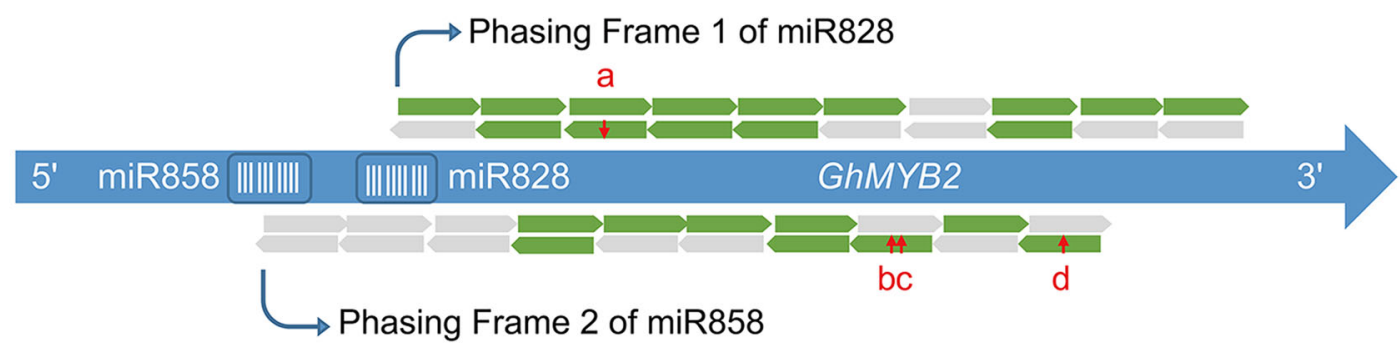 \\ phasiRNA shown in small RNA seq libraries \\ phasiRNA absent in small RNA seq libraries \\ b \\ AACUUCCUCCUCGGAACAAAU PF1_3' D3(-)
a $(2 / 35)$ \\ UAAAAGGAGUGAUGAAAUUG PF2_3' D8(-) $\stackrel{4}{\mathrm{C}}(23 / 35)$ \\ UAAAAGGAAGUGAUGAAAUUG PF2_3' D8(-) b $(9 / 35)$ \\ AUGUGGACGAAUUCUUUGACU PF2_3' D10(-) \\ Fig. 2 Cis-effects of phasiRNA derived from GhMYB2 RNA in anther, hypocotyl and EC tissues. a, Schematic phasing model for the hypothesis that there is a phasing frame 1 (PF1) of the miR828 3' cleavage site and phasing frame 2 (PF2) of the miR858 3' cleavage site. The red arrows and letters represent the GhMYB2 mRNA cleavage sites detected by RLM RACE. $\mathbf{b}$, The sequences of each cleavage sites were enlarged. The frequency of each cleavage sites were shown. Out of 35 cleavage events detected, 2, 9, 23 and 1 events were on site a, b, c and d respectively. The detected cleavage sites were on PF1_3'D3(-), PF2_3'D8(-) and PF2_3'D10(-)}

Tissue-specific expression of phasiRNAs at GhMYB2 loci In order to determine whether phasiRNA generation is associated with the expression of the origin gene, we examined the expression of the two miRNA precursors and GhMYB2 in cotton ovules, anthers, hypocotyls, and EC tissue using qRT-PCR. Results indicated that, the expression levels of GhmiR828 and GhmiR858 precursors were high in ovules at 0 and 3 days post anthesis (DPA), hypocotyls, but low in early ovules at -3 DPA, anthers and EC tissues (Fig. 3a, Supplemental table 6 and Supplemental fig. 3). The GhMYB2 expression level was highest in hypocotyl tissues and lowest in early ovules of - 3 DPA, anthers and EC tissues, with levels in early ovules of 0 and 3 DPA in between (Fig. 3a). The abundant accumulation of miR828 and miR858 at ovules of 0 DPA and 3 DPA was consistent with previous discovery [24]. Notably, the 3 DPA ovules with relatively higher miR828 and miR858 levels when compare with 0 DPA ovules, showed relatively lower GhMYB2 mRNA expression, indicating the precise regulation of GhMYB2 genes by miRNA during cotton fibre development.

\section{The molecular basis of tissue-specific expression of GhMYB2 derived phasiRNAs}

The cotton genome underwent multiple rounds of whole genome duplication. We speculate that cotton genome carries redundant proteins in phasiRNA biogenesis. To test this hypothesis, we examined the endogenous expression of AGO1, SGS3, RDR6 and DCL4 in cotton ovules, anthers, hypocotyls, and EC tissue using both mRNA sequencing data and qRT-PCR. We discovered that genes responsible for phasiRNA biogenesis were actively expressed in early ovule tissues, which corresponds to small RNA activity in fertilized ovules (Fig. 3). The expression of these genes was lower in anther tissues. AGO1 expression was comparable in ovule and hypocotyl tissues, but RDR6 and DCL4 expression was significantly higher in hypocotyls than in other tissues (Fig. 3b). All of these gene expression patterns agree with the high abundance of phasiRNAs derived from GhMYB2 in hypocotyls (Fig. 3a). While the absence of phasiRNA biogenesis in EC tissue corresponded to the undetectable levels of GhphasiRNA1 and GhMYB2 phasiRNAs in undifferentiated EC tissues. In the reproductive tissues, $A G O 1$ expression was very active (Fig. $3 \mathrm{~b}$ ), a pattern similar to that observed in rice [32], which we noted earlier. The expression of $D C L$ genes was also higher in hypocotyl tissue than in anther tissue (Fig. 3b). We know that in Arabidopsis, the function of DCL4 can be carried out by DCL1. But unlike the 21-nt final products of $D C L 4, D C L 1$ generates more 22-nt siRNA [33]. In this same way, GhphasiRNA1, and GhMYB2 in the cotton genome generated not only 21-nt phasiRNAs, but they can also generate 18$29 \mathrm{nt}$ siRNAs (Supplemental dataset 1). To unveil the potential redundant functions of phasiRNA-biogenesis proteins in cotton, we extended the corresponding homologs in genome wide. The expression patterns of 
a
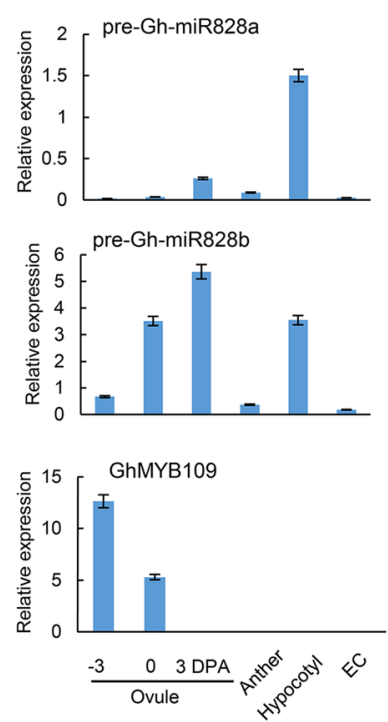

b
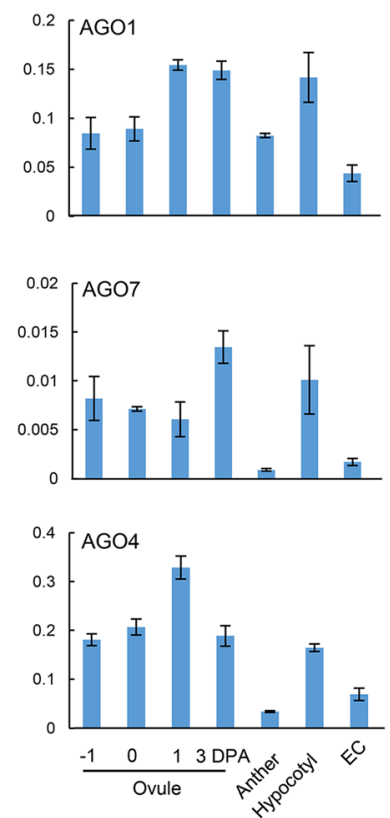
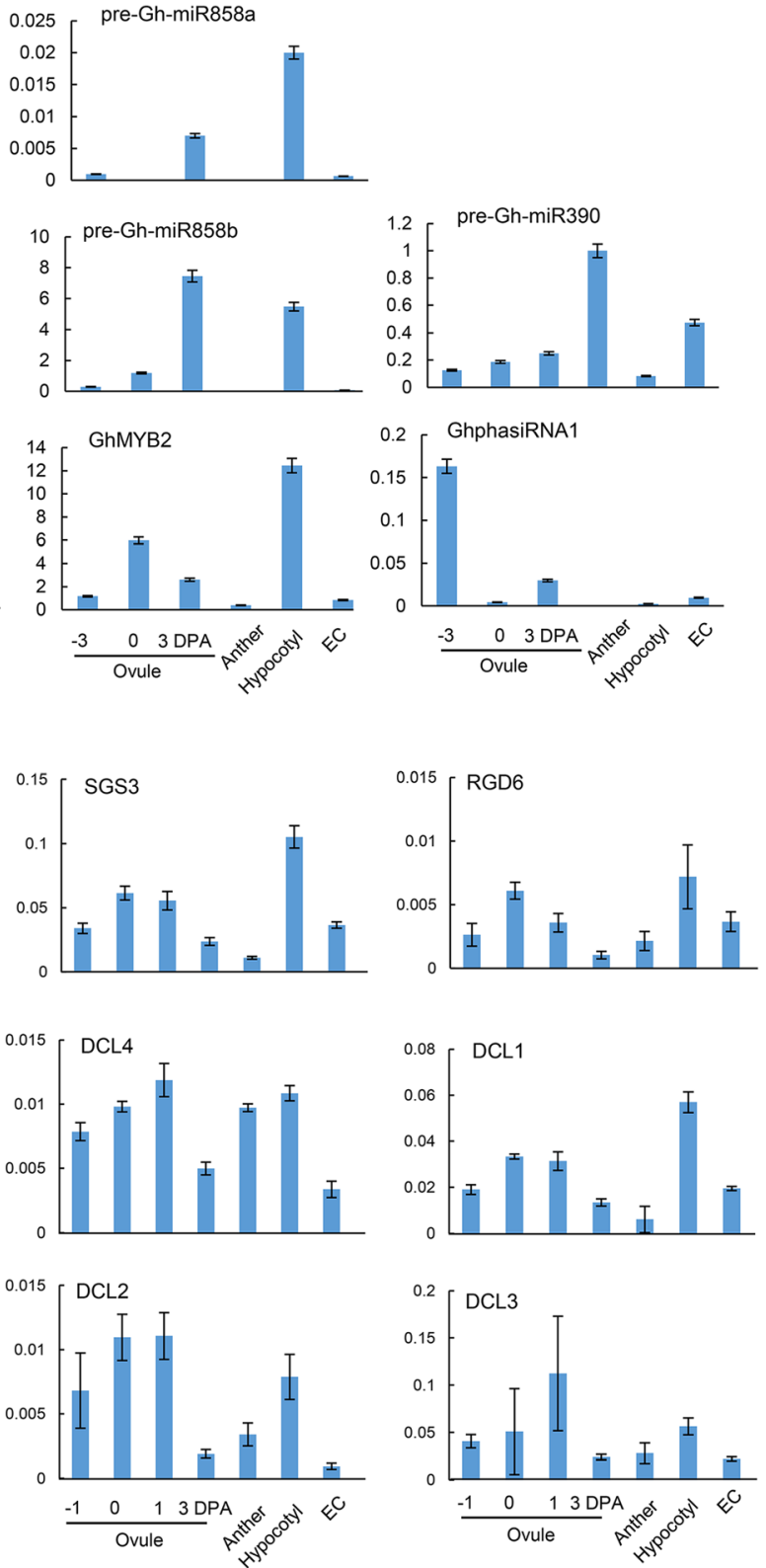

Fig. 3 Expression pattern of phasing RNA triggering miRNA, their targeting genes and genes involved in tasiRNA biogenesis. $\mathbf{a}$, qRT-PCR analysis of precursor forms of miRNAs including GhmiR828, GhmiR858 and their targeting gene GhMYB2. GhmiR390 and its targeting gene GhphasiRNA1 was served as the positive controls for phasiRNA generation. GhMYB109 was miR828 targeting gene and used as negative control for phasiRNA deriving. b, qRT-PCR analysis of genes involved in tasiRNA biogenesis, including AGO1, SGS3, RDR6, DCL4. The genes of AGO4, AGO7, DCL1, DCL2 and DCL3 were used as internal controls for gene redundancy in early ovules from - 1, 0, 1 and 3 days post anthesis (DPA), anthers, hypocotyls and seeds. Three biological replicates were used for each tissue

AGO, RDR6, SGS and DCL homologs were extensively examined via mRNA-seq analysis in a selection of 14 cotton tissues. These genes showed a distinct tissue specific pattern in general which was similar to the pattern shown with the qRT-PCR analysis (Supplemental fig. 2, Supplemental table 6). These results excluded the potential error introduced by the internal control gene selection of qRT-PCR. Therefore, AGO1 might lead the slicing of miRNA/mRNA, but DCL1 and DCL4 likely carry out the final cleavage of dsRNA. The proactive expression activity of Argonaute and DCL coding genes in somatic tissues than undifferentiated cell might be responsible for the abundant expression of phasiRNAs. 
GhMYB2 loci in the cotton genome generate phasiRNAs in somatic tissues, but not in undifferentiated cells

GhMYB2 is a coding gene known to generate phasiRNAs in the fiber-bearing tissues of cotton [24]. Both the phasiRNA trigger, miR858 and miR828 and the GhMYB2 gene expression showed tissue-specific pattern is consistent with the GhMYB2 function in fiber cell regulation. In order to detect GhMYB2 loci capability of generating phasiRNAs in a variety of tissues, we investigated potential phasiRNA-deriving genes in the cotton genome. Our previous studies predicted that GhMYB109 is targeted by miR828 to generate phasiRNAs. However, small RNA mapping and miRNA cleaving test has suggested that GhMYB109 is not in fact a phasiRNAderiving gene in cotton. Consequently, in this study GhMYB109 was employed as a negative control for phasiRNA generation. We used the reverse complementary rule of miRNA targeting to predict the target sites of miR173 and miR390 in the Upland cotton genome (G. hirsutum, TM-1), allowing three mis-matches and one $\mathrm{T}-\mathrm{G}$ bulge. We then identified 45 of the predicted miR173 targets and 13 of the predicted miR390 targets as potential phasiRNA-deriving loci in the cotton genome (Supplemental table 3). GhphasiRNA1 presented a particular abundance of small RNA transcripts in early ovule, anther, hypocotyl and EC (Fig. 4). Therefore, they were selected as positive controls for phasiRNA-deriving cotton genes. Finally, the cotton GhUBL1 gene, which encodes ubiquitin, was selected as the non-miRNA targeting control.

Small RNA density plot used the perfect mapped reads from each sample. To compensate the lacking of biological replication from each tissues sample, we selected three somatic tissues, early ovule, anther and hypocotyl as independent biological samples compared with the undifferentiated sample, EC tissue (Supplemental table 2).

We observed that GhMYB2 loci from both the A and $D$ subgenomes of cotton generated high densities of small RNA distribution in the ovule, anther and hypocotyl (Fig. 4), similar to GhphasiRNA1. Because the low polymorphism between the A and D alleles, GhMYB2 small RNA density didn't exhibit obvious differences allelically (Fig. 4a). As expected, GhMYB109, in a pattern similar to that of GhUBL1, did not generate high levels of small RNA in any tissues (Fig. 4). In addition, we noted that neither GhMYB2 nor GhphasiRNA1 generated much siRNA in the embryogenic callus (EC) tissue, as shown in Fig. 4b. These results suggest that GhMYB2 can express and produce phasiRNAs in differentiated

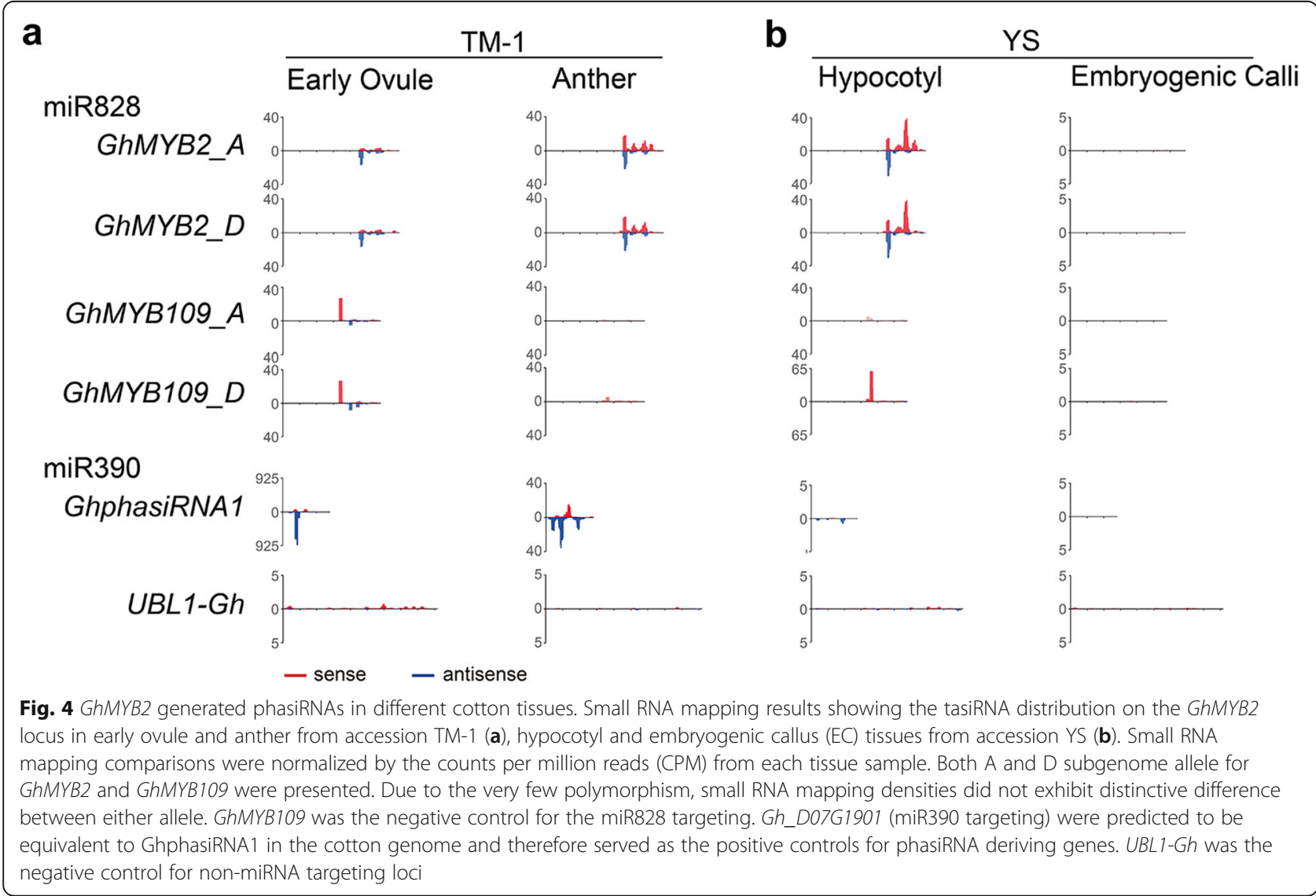


somatic cells but not in undifferentiated EC cells due to the tissue-specific expression pattern.

\section{GhMYB2-derived phasiRNAs take potential roles in trans- regulate downstream targets}

To further examine the potential functions of GhMYB2derived phasiRNAs, we predicted the phasiRNA targets in the cotton genome according to the reverse complementary rule, allowing 2 mismatches (Supplemental dataset 2). To avoid the effects of random small RNA, only the overlapping phasiRNAs from ovules, anthers and hypocotyls were selected for the target predicting investigation. The expression of phasiRNA targets in cotton ovules, anthers and hypocotyl tissue are presented in the heatmap in Fig. 5a. The differentially expressed genes (DEGs) of phasiRNA target genes in anthers and hypocotyls are shown in a VENN diagram in Fig. 5b. Only 41.3\% (38/92) and 63.3\% (38/60) of all target genes were common, up-regulated genes in hypocotyls and anthers, respectively. However, 97.2\% (65/67) of down-regulated target genes in hypocotyls overlapped with those in anthers. The down regulated target genes in anthers were significantly enriched in hypocotyls (Fisher's exact test, $P<6.54 \mathrm{e}^{-15}$ ). The high proportion of

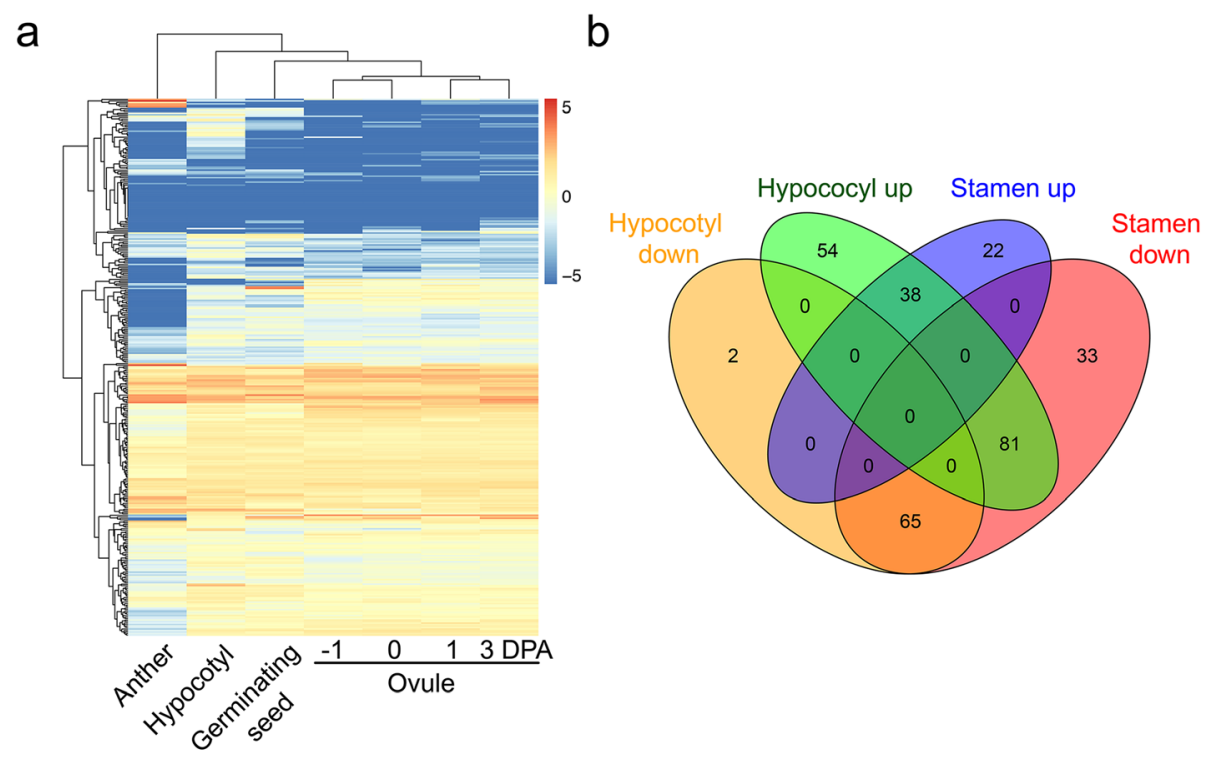

C

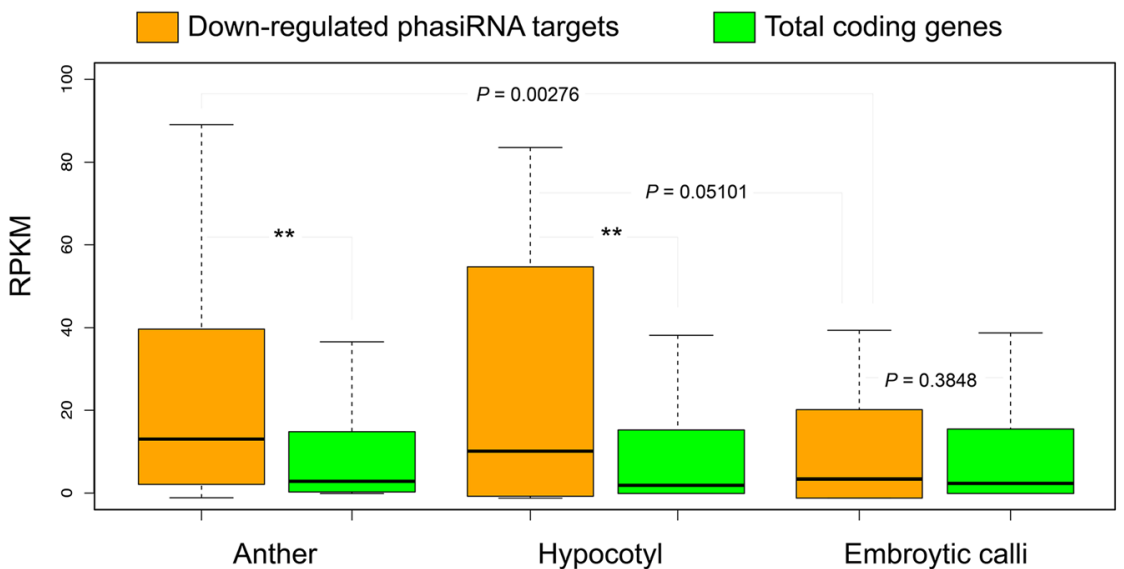

Fig. 5 phasiRNA target gene expression profile. a: Heat map displaying the expression pattern of the predicted phasiRNA targets in the cotton genome. PhasiRNAs were derived from GhMYB2 loci. Only conserved tasiRNAs that were consistently expressed in ovule, anther, hypocotyl tissues were selected. The phasiRNA targets were clustered according to their tissue expression patterns. $\mathbf{b}$ : Venn diagram illustrating differences between the expression patterns of the predicted phasiRNA target genes. All comparisons were against to the early ovule tissues. $\mathbf{c}$, Degradome analysis of the down-regulated phasiRNA targets in anther, hypocotyl and EC tissues. Box plot showing the abundance of degraded fragments in anther, hypocotyl and EC tissues using RPKM values. The central line for each box plot indicates the median. The yellow represents the population of down-regulated phasiRNA targets in anther and hypocotyls tissue. The total levels of degraded fragments of coding genes were used as a background control (green). The $P$ value was calculated using the Wilcoxon rank sum test 
common, down-regulated genes in anthers and hypocotyls suggests that the phasiRNAs in these tissues target similar genes in similar functional pathways. GO enrichment analysis indicated that these phasiRNA targets were involved in cytoskeletal anchoring at plasma membrane at high Rich Factor (Supplemental Fig. 4). The cell skeleton arrangement is related to the structure of cell shape. We found that the enrichment of downregulating genes in this category reflects the unique function of GhMYB2 as a phasiRNA-deriving gene in specific cell morphology, especially in fiber cells (Supplemental table 4).

Anthers were highly enriched with 21- and 24-nt phasiRNAs [16]. In order to examine whether the suppression of phasiRNAs target expression in anthers and hypocotyls is due to GhMYB2-derived phasiRNA, we compared the degraded fragments of the 65 phasiRNA targets to the total genome. To avoid the effects of random degraded fragments, the reads mapped on $5^{\prime}$ end of the phasiRNAs targeting sites were filtered out. The median level of degradation of the 65 phasiRNA targets in anthers and hypocotyls was twice that of the total genome (Fig. 5c) (Wilcoxon rank sum tests, anther vs EC $P=0.0276$; hypocotyl vs EC $P=0.05101$ ). The phasiRNA targets degraded fragments were significantly higher than the total coding gene background in anther, hypocotyl and EC tissues (Wilcoxon rank sum tests, typically $\left.P<2.2 \mathrm{e}^{-16}\right)$. Therefore, the differences in expression of the 65 phasiRNA target genes might be caused by phasiRNA trans- regulation. This suggested that the phasiRNAs generated by GhMBY2 in cotton could down-regulate target gene expression by increasing the degradation of their mRNA.

\section{Discussion}

\section{GhMYB2 has two phasing frames for phasiRNAs} production in the cotton genome

PhasiRNAs is a unique type of small RNA. It can be derived from trigger miRNAs with a length of $21 \mathrm{nt}$ or 22 nt [34]. In the past decade, several phasiRNA triggers have been identified in moss [35], olives [36], chinese fir [37], apple [20], rice [38], cotton [27, 39], sorghum [40], Dimocarpus longan Lour [41] and soybean [42]. One model of phasiRNA triggering consists of a 'one-hit' system that usually involves a 22-nt microRNA triggers. MiR173 is such conserved phasiRNA trigger in plant genomes. Another model of phasiRNA biogenesis consists of a 'two-hit' system, which uses two 21-nt microRNAs per mRNAs transcript [34]. Representative TAS genes of 'two-hit' model carry two miR390 targeting sites found in moss and Arabidopsis [7]. These mRNAs carry two miR390 reverse complementary sites across a 200-300 nt region. PhasiRNAs are found to be active in the region between the two miRNA targeting sites in phase of the $5^{\prime}$ to the cleavage site of miR390. Alternative phasing in this region has also been reported [7]. There are two hypotheses for the cause of this alternative phasing: cis- activation of one of the phasiRNAs, or activation of the $5^{\prime}$ miR390 cleavage site.

Some of the miR828 targeting loci in apple and cotton genomes have been reported to be targeted by two miRNAs; miR828 and miR858 [20, 24]. Two-hit GhMYB2 can generate phasiRNAs in phase of the 3' miR828 cleavage site [20, 24]. In our present research, we observed that the GhMYB2 loci actively generated phasiRNAs in the alternative phasing frame of the 3' miR858 cleavage site and that GhMYB2 can generate phasiRNAs on both PF1 and PF2 in multiple tissues.

Due to the existence of a double phasing frame for the loci, the phasing score curve for GhMYB2 shows double peaks on each phase. This is not a typical phasing curve. Alternative phased small RNAs have never been considered in phasiRNAs analysis. Therefore, the conventional evaluation of phasiRNA prediction, which follows the principle of one phasing frame on one locus, could underestimate the amount of phasiRNAs in the genome. This revelation could in turn impact the analysis of the function of phasiRNAs.

\section{Tissue-specificity of phasiRNA expression in cotton}

The abundance of phasiRNAs of origin loci vary from tissue to tissue. GhphasiRNA1 generates phasiRNAs in reproductive tissues (Fig. 4) and GhMYB2 produces more phasiRNAs in vegetative tissues than in ovules (Fig. 4). It is likely, then, that the efficiency of phasiRNAs biogenesis differs between tissues.

The biogenesis of phasiRNAs requires a unique set of proteins, but collective data reveal the functional redundancy of the involved Argonaute and DCL protein families. In Arabidopsis, an Argonaute protein is thought to be required for the slicing of 22-nt miRNA-targeted mRNA [43]. In a recent report, those derived 21-nt phasiRNA was detected in an ago1 null mutant [43]. The assimilated impacts of $\mathrm{AGO} 2$ and AGO7 in phasiRNA biogenesis $[6,8,44]$ are redundant within the Argonaute protein family. Similarly, dicer-like proteins DCL1-4 digest dsRNA into small RNA [4] while several different DCL proteins carry out functions related to miRNA, siRNA and phasiRNAs. DCL4 specifically recognizes RDR6-generated double stranded RNA and cuts it into 21 nt RNA [2]. But in the absence of DCL4, this function is adopted by DCL1 with the product of phased small RNAs in length of $22 \mathrm{nt}$ [33].

We observed the expression pattern of phasiRNA biogenesis related genes in cotton tissues. These results suggest that the phase-change of phasiRNAs loci is primarily dependent on the activity of Argonaute and Dicer-like proteins, which varies among tissues. The 
activity of $D C L 1, D C L 2, D C L 3$ and $D C L 4$ expression was consistently high in early ovule and fiber tissues (Fig. 3b). RDR6 and DCL4 mRNA expression pattern was identical in hypocotyls. DCL1, DCL2 and DCL3 were all found to be actively expressed in the hypocotyl (Fig. 3b). The expression of AGO1, AGO4 and AGO7 mRNA was also high in hypocotyls (Fig. 3b). Therefore, the redundant Argnaute and DCLs most likely slice the long dsRNA into small RNAs of different lengths and phases.

\section{The cis- function of phasiRNAs}

PhasiRNAs is common in plant genomes, but little has been determined about the biological functions of these small RNAs. In 2014, Small RNA sequencing of maize anthers at different developmental stages led to the discovery that phasiRNA expression is associated with the meiosis progress $[15,16]$. The expression of 21 -nt phasiRNAs occurs prior to that of 24-nt phasiRNAs in anther development, which suggests that 21-nt phasiRNAs and 24-nt phasiRNAs play different roles in meiosis. Analysis of maize small RNA also revealed that phasiRNAs are the dominant small RNA population in anther tissue [16]. These findings began to elucidate the specific functions of 21-nt phasiRNAs. However, the precise function of these phasiRNAs has remained unknown.

In response, we tried to argue through RNA degradation data analysis that phasiRNA might play a role in
mRNA degradation by cis-regulation (Fig. 6). The original phasiRNA location could be the primary target site of phasiRNAs, especially in anthers. Degraded fragments of GhMYB2 mRNA are enriched in those tissues with high levels of phasiRNA generation. This phenomenon would seem to indicate that phasiRNA targets the original mRNA in a similar way to miRNA, in order to guide mRNA cleavage. However, we also found that the degraded fragments were not enriched in hypocotyl tissue, though phasiRNAs were expressed. Therefore, mRNA degradation is tissue-specific. This ultimately suggests that mRNA degradation must be under the control of other unknown elements in the genome beyond small RNA.

\section{The trans- effect of phasiRNAs on gene silencing}

Small RNA plays unique roles in fiber development, and its process could be mirrored in phasiRNA function. The small RNA profiles of leaves and ovules have been found to be distinctly different in the early stages of fiber development and during and after fertilization [45]. Fuzz fiber differentiation is determined by the $N_{1}$ gene, GhMYB25-like/GhMML3 [46]; the suppression of fuzz is caused by small RNAs derived from the GhMML3 locus. The small RNA from GhMML3 in $N_{1}$ is generated from dsRNA formed by the sense and antisense transcripts of GhMML3.

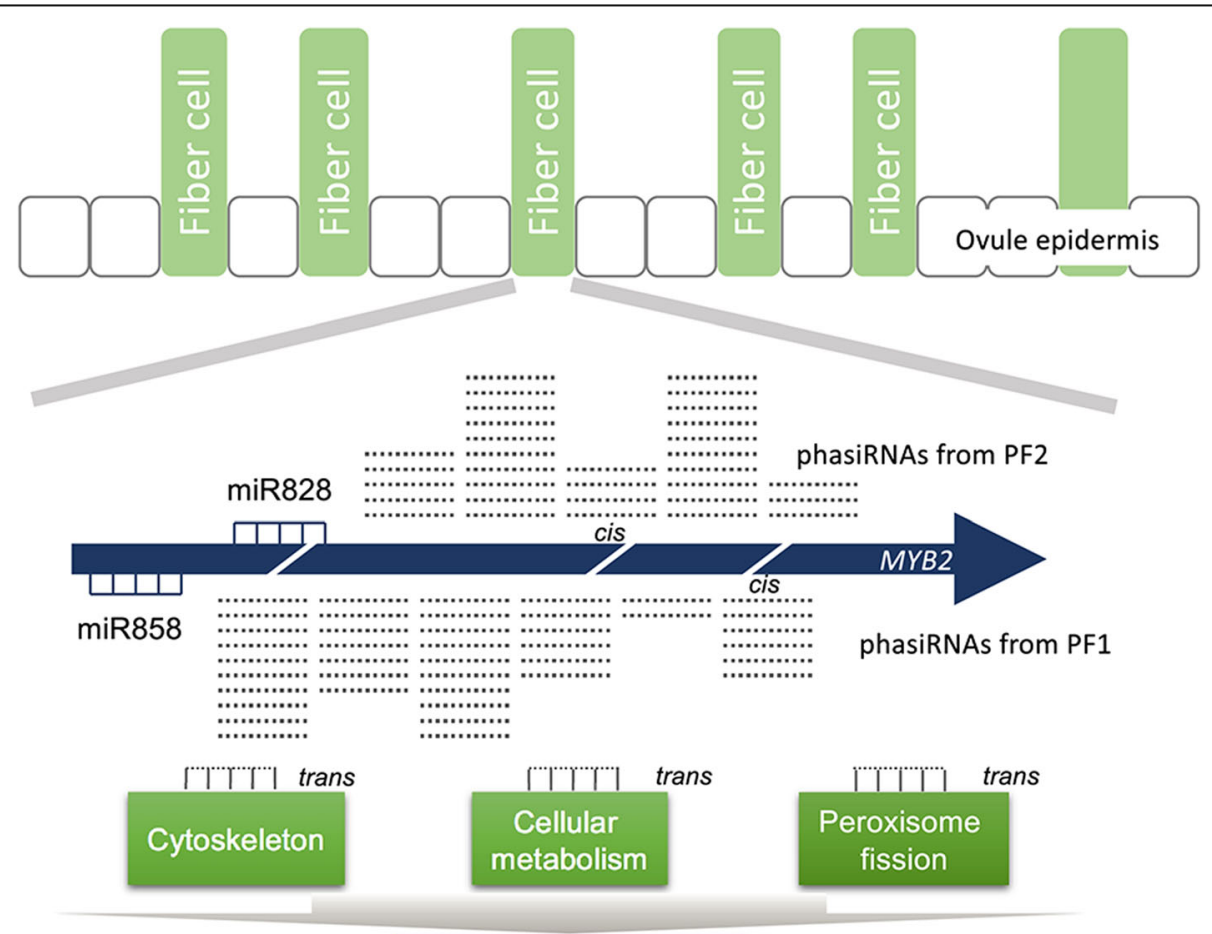

Fiber cell development

Fig. 6 The working model of phasiRNA derived from MYB2 locus in cis- and trans- regulation 
Although the biogenesis pathway is different, phasiRNAs could affect the genome in a way similar to small RNA from Natural antisense transcripts (NATs) [47]. Collective data suggest that NAT-derived small RNAs are involved in de novo DNA methylation and histone modification [46]. These small RNAs could follow the reverse complementary base pair rules to find the deductive target location with a relatively high accuracy. Herein we predicted the phasiRNA targets in the cotton genome, which appear to be suppressed in hypocotyls. The hypocotyl tissue was found to have the highest phasiRNA production from the GhMYB2 locus out of all examined tissues. The suppression of these genes in the hypocotyl tissue suggests a correlation between phasiRNAs and its potential targets, which would mean that phasiRNAs could play a role in trans-gene regulation (Fig. 6).

\section{Conclusions}

In this study, we have extensively analyzed the phasing pattern and function of GhMYB2-derived phasiRNAs. We integrated small RNA seq, mRNA seq and mRNA degradome data to investigate the behavior and functions of phasiRNA in the cotton genome. Using cotton GhMYB2, TAS1/2, TAS3 as research examples, we found that $G h M Y B 2$ can generate phasiRNAs on two phasing frames; one at the $3^{\prime}$ cleaved end of miR828 and one at the miR858 target site. The known two-hit trigger of TAS by two miR390 sites only presents one phasing frame. This one-phasing frame principle for phasiRNA biogenesis inspired a huge amount of genome-wide phasiRNA examination in Arabidopsis, rice, maize and many other species. Our novel finding of an alternative phasing frame on one locus may modify the formula for phasiRNA prediction and evaluation. PhasiRNAs can cis- regulate GhMYB2 mRNA by degrading it. In our study, we provide the direct evidence of the endogenous functions of phasiRNA in a plant genome.

\section{Methods \\ Cotton material growth conditions and cotton tissue collection}

Upland cotton (Gossypium hirsutum) standard accession Texas Marker-1 (TM-1) was obtained from Crop Research Division, ARS, USDA, College Station, Texas. TM-1 was grown in the greenhouse with a $28^{\circ} \mathrm{C}, 16 / 8 \mathrm{~h}$ (light/dark) light cycle. Ovule-bearing fibers on the epidermis and anther were harvested on the day of flowering (0 days-post-anthesis, DPA) for small RNA sequencing sampling. Hypocotyl tissues were harvested from 4-week old cotton seedlings. Embryogenic calli tissues were harvested from calli grown from hypocotyls tissues in embryogenic media for 8 weeks. At least three biological replications were used for each tissue. Ovules, hypocotyl, anther and embryogenic calli tissues were harvested for RNA extraction and qRT-PCR validation.

\section{Small RNA sequencing, degradome and cotton mRNA sequencing data sources}

Small RNA sequencing data for cotton ovules from Gossypium hirsutum, acc. TM-1 were previously produced in our lab and published in The National Center for Biotechnology Information, Gene Expression Omnibus (NCBI GEO, https://www.ncbi.nlm.nih.gov/geo/) with accession number of PRJNA293171, TM-1 small RNA accession SRX1174194. The RNAs from - 1, 0, 1, 3 and 5 DPA ovule tissues were mixed to prepare the small RNA library of early ovule. Small RNA sequencing data for G. hirsutum anthers, hypocotyls and embryogenic calli were downloaded from NCBI GEO, accession numbers GSE43531 (anther, small RNA and degradome) and GSE41132 (hypocotyl and embryogenic calli, small RNA and degradome) [48]. The reference genome was the Upland cotton TM-1 genome [49].

\section{RNA extraction and real-time quantitative reverse transcription PCR (qRT-PCR)}

RNA extraction was conducted using the Plant RNA Express Extraction kit (RK2002, Zoonbio Biotechnology) and RNA quality was examined by Nanodrop. Reverse transcription was conducted using the first strand cDNA synthesis kit (PC24-50 T, Zoonbio Biotechnology) and qRT-PCR was performed on an ABI 7500. The relative gene expression was normalized to the cotton Histone 3 gene [46] using the $\Delta \Delta C$ t method [50]. The qRT-PCR primer set was designed as described [51]. All gene accession number and qRT-PCR primer information are listed in supplemental table 5. MiRNA precursors were listed in supplemental table 6.

\section{Bioinformatics analysis of small RNA mapping and degradome mapping}

Raw reads of small RNA and degradome sequencing libraries were obtained from NCBI GEO published database, representing three type of somatic tissues of ovule, anther and hypocotyl, and one undifferentiated tissue, embryogenic calli. Because lacking of plenty biological replicates for each sample, three different somatic tissues were employed as independent biological samples for comparison. After adapter clipping, the clean reads from each library were mapped to the candidate genes using Bowtie [52] (settings -a -v 0), allowing no mismatches and retaining all alignments. The retained sRNA reads were $14,956,687$ for early ovule, $16,347,976$ for anther, 24,948,581 for hypocotyl and 25,984,975 for EC (Supplemental table 2). The small RNA comparisons among tissue samples were normalized by counts per million (CPM) value on the same phasiRNA deriving locus. The 
small RNA total mapping rate was 90-93\% (Supplemental table 2). The unique mapping rate and multimapping rate were all above $40 \%$ for each library (Supplemental table 2), representing a fine small RNA profile for further study. The small RNA densities were normalized by counts per million (CPM) value.

The degradome qualifications were examined with Ylab: RPKM. The central line for each box plot indicates the median. The top and bottom edges of the box indicate the 25th and 75th percentiles and the whiskers extend 1.5 times the interquartile range beyond the edges of the box. Significance was evaluated using Wilcoxon rank sum tests.

\section{Mapping of the MYB2 mRNA internal cleavage sites by phasiRNAs}

For mapping the internal cleavage site in $M Y B 2$ mRNA, RNA ligase-mediated rapid amplification of cDNA ends (RLM-RACE) was done using the FirstChoice ${ }^{\bullet}$ RLMRACE Kit (Invitrogen). Total RNA was isolated from the tissues used as indicated in the results, include anther, hypocotyl, ovules and EC. Total RNA $(4 \mu \mathrm{g})$ was directly ligated to the GeneRacer RNA Oligo adapter without the treatment of calf intestine alkaline phosphatase (CIP) and tobacco acid pyrophosphatase (TAP). Outer 5' RLM-RACE PCR was performed using the 5' RACE Outer Primer (5'-GCTGATGGCGATGAATGAACAC TG-3') and 5'RACE gene specific outer primer (5' -TACCATTGCTAATGGATCCTGTTGAT-3') to prime first strand cDNA synthesis in reverse transcription reaction. Inner 5' RLM-RACE PCR was then applied using 5'RACE inner primer (5'-CGCGGATCCGAACACTGC GTTTGCTGGCTTTGATG-3') and 5'RACE gene specific innner primer $\left(5^{\prime}\right.$-CATCAAGTTCAAGGAACT TATTCACC- $\left.3^{\prime}\right)$. PCR products were gel purified and cloned into pGEM-T Easy vector (Promega, Madison, WI) for sequencing.

\section{Phase transformation evaluation}

Phase transformation data were produced using the following optimized equation [31]:

$$
P=\ln \left[\left(1+\sum_{i=1}^{10} k i\right)^{n-2}\right], P>0 .
$$

where $\mathrm{n}$ is number of phase cycle positions occupied by at least one small RNA read within an eight-cycle window, and $\mathrm{k}$ is the total number of reads for all small RNAs with consolidated sequence in a given phase within an eight-cycle window. The cutoff of the phasing score was arbitrarily set at 5 .

\section{PhasiRNA target prediction}

Based on the phasiRNAs from GhMYB2A and GhMYB2D in all ovules, anthers, hypocotyls and embryogenic calli as the conserved phasiRNA query, the phasiRNA targets in the Upland cotton TM-1 genome were predicted [49]. The method of phasiRNA target prediction was that of the TargetFinder (https://github. com/carringtonlab/TargetFinder) online tools. The parameters were set to a TargetFinder score of $\geq 140$ and a small RNA::mRNA interaction secondary structure maximum free energy (MFE) of $\leq-20 \mathrm{kcal} / \mathrm{mol}$.

\section{Supplementary information}

Supplementary information accompanies this paper at https://doi.org/10. 1186/s12870-020-02430-3.

Additional file 1: Figure S1. The secondary structure of miR828 and miR858 precursors

Additional file 2: Figure S2. The heatmap of mRNA activity showing the AGO, DCL, RDR6, SGS3 homologs in upland cotton genome based upon mRNA seq analysis

Additional file 3: Figure S3. Distribution of degraded fragments of GhMYB2 mRNA. GhMYB109 was used as the negative control for the miR828 targeting. GhphasiRNA1 was predicted to be phasiRNA deriving genes in the cotton genome to serve as positive control. UBL1-Gh was used as the negative control for non-miRNA targeting loci. PhasiRNA may play a role in mRNA degradation at its origin loci, as shown in the anther degradome data. They may also play roles in other target sites, as shown in the hypocotyl degradome distribution and heat map

Additional file 4: Figure S4. Functional enrichment analysis of the down-regulated gene group from Fig. 5 panel $b$ in both hypocotyl and anther tissues

Additional file 5: Dataset 1

Additional file 6: Dataset 2

Additional file 7: Table S1. phasiRNA read number in small RNA libraries from two phasing frames on GhMYB2

Additional file 8: Table S2. The mapping statistics for the small RNA libraries and degradome libraries used in this study

Additional file 9: Table S3. The miR173 and miR390 predicted target in cotton genome

Additional file 10: Table S4.

Additional file 11: Table S5. Gene accession and primer

Additional file 12: Table S6. The sequences of miRNA precursors

\section{Abbreviations}

phasiRNA: Phased small interfering RNA; RLM-RACE: RNA ligase-mediated rapid amplification of CDNA ends; phasiRNA: Trans-acting small interfering RNA; EC: Embryogenic calli; PF: Phasing frame; DPA: Days post anthesis; DEGs: Differentially expressed genes

Acknowledgements

Not applicable

Authors' contributions

$X$. G. conceptualized the research program, designed experiments and coordinated the project. X. G., T. Z., X.T. and J. C. analyzed all data and wrote the manuscript. X.T., M. G., M. L., N. Z., J.C., G. M., J. L., K. W. and L. D. conducted laboratory experiments and were involved in data analysis. L.F., J. C. B. Z. and T. Z. provided the mRNA-seq data. All authors discussed results and commented on the manuscript. The author(s) read and approved the final manuscript. 


\section{Funding}

This work was financially supported in part by grants from the National Key Research and Development Program of China (2016YFD0101006), the National Natural Science Foundation of China (NSFC, 31971985, 31900395), China Postdoctoral Science Foundation (2018 M632477, 2019 M652094), the Fundamental Research Funds for the Central Universities, the JCIC-MCP project, Dabeinong Funds for Discipline Development and Talent Training in Zhejiang University. All these grants support in the design of the study and collection, analysis, and interpretation of data and in writing the manuscript.

\section{Availability of data and materials}

The datasets used and analyzed during the current study available at The National Center for Biotechnology Information, Gene Expression Omnibus (NCBI GEO, https://www.ncbi.nlm.nih.gov/geo/) with accession number of PRJNA293171, GSE43531 (anther, small RNA and degradome) and GSE41132 (hypocotyl and embryogenic calli, small RNA and degradome). The plant materials are available from the corresponding author on reasonable request.

\section{Ethics approval and consent to participate}

The experimental research on plants performed in this study complies with institutional, national and international guidelines.

\section{Consent for publication}

Not applicable

\section{Competing interests}

The authors declare that they have no competing interests.

Received: 27 December 2019 Accepted: 5 May 2020

Published online: 15 May 2020

\section{References}

1. Zheng Y, Wang Y, Wu J, Ding B, Fei Z. A dynamic evolutionary and functional landscape of plant phased small interfering RNAs. BMC Biol. 2015; 13:32

2. Felippes FF, Weigel D. Triggering the formation of tasiRNAs in Arabidopsis thaliana: the role of microRNA miR173. EMBO Rep. 2009;10(3):264-70.

3. Vazquez F, Vaucheret H, Rajagopalan R, Lepers C, Gasciolli V, Mallory AC, Hilbert J-L, Bartel DP, Crété $P$. Endogenous trans-acting siRNAs regulate the accumulation of Arabidopsis mRNAs. Mol Cell. 2004;16(1):69-79.

4. Xie Z, Allen E, Wilken A, Carrington JC. DICER-LIKE 4 functions in transacting small interfering RNA biogenesis and vegetative phase change in Arabidopsis thaliana. Proc Natl Acad Sci U S A. 2005;102(36):12984-9.

5. Allen E, Xie Z, Gustafson AM, Carrington JC. microRNA-directed phasing during trans-acting siRNA biogenesis in plants. Cell. 2005;121(2):207-21.

6. Montgomery TA, Yoo SJ, Fahlgren N, Gilbert SD, Howell MD, Sullivan CM, Alexander A, Nguyen G, Allen E, Ahn JH, et al. AGO1-miR173 complex initiates phased siRNA formation in plants. Proc Natl Acad Sci U S A. 2008; 105(51):20055-62.

7. Axtell MJ, Jan C, Rajagopalan R, Bartel DP. A two-hit trigger for siRNA biogenesis in plants. Cell. 2006;127(3):565-77.

8. Montgomery TA, Howell MD, Cuperus JT, Li D, Hansen JE, Alexander AL, Chapman EJ, Fahlgren N, Allen E, Carrington JC. Specificity of ARGONAUTE7-miR390 interaction and dual functionality in TAS3 transacting siRNA formation. Cell. 2008;133(1):128-41.

9. Cuperus JT, Montgomery TA, Fahlgren N, Burke RT, Townsend T, Sullivan CM, Carrington JC. Identification of MIR390a precursor processing-defective mutants in Arabidopsis by direct genome sequencing. Proc Natl Acad Sci U S A. 2010;107(1):466-71.

10. Hsieh LC, Lin SI, Shih AC, Chen JW, Lin WY, Tseng CY, Li WH, Chiou TJ. Uncovering small RNA-mediated responses to phosphate deficiency in Arabidopsis by deep sequencing. Plant Physiol. 2009;151(4):2120-32.

11. Chen HM, Chen LT, Patel K, Li YH, Baulcombe DC, Wu SH. 22-nucleotide RNAs trigger secondary siRNA biogenesis in plants. Proc Natl Acad Sci U S A. 2010;107(34):15269-74.

12. Cuperus JT, Carbonell A, Fahlgren N, Garcia-Ruiz H, Burke RT, Takeda A, Sullivan CM, Gilbert SD, Montgomery TA, Carrington JC. Unique functionality of 22-nt miRNAs in triggering RDR6-dependent siRNA biogenesis from target transcripts in Arabidopsis. Nat Struct Mol Biol. 2010; 17(8):997-1003.
13. Luo QJ, Mittal A, Jia F, Rock CD: An autoregulatory feedback loop involving PAP1 and TAS4 in response to sugars in Arabidopsis. Plant molecularbiology 2012, 80(1):117-129.

14. De Paoli E, Dorantes-Acosta A, Zhai J, Accerbi M, Jeong DH, Park S, Meyers BC, Jorgensen RA, Green PJ. Distinct extremely abundant siRNAs associated with cosuppression in petunia. RNA (New York, NY). 2009; 15(11):1965-70

15. Dukowic-Schulze S, Sundararajan A, Ramaraj T, Kianian S, Pawlowski WP, Mudge J, Chen C. Novel meiotic miRNAs and indications for a role of PhasiRNAs in meiosis. Front Plant Sci. 2016;7:762.

16. Zhai J, Zhang H, Arikit S, Huang K, Nan GL, Walbot V, Meyers BC. Spatiotemporally dynamic, cell-type-dependent premeiotic and meiotic phasiRNAs in maize anthers. Proc Natl Acad Sci U S A. 2015;112(10):3146-51.

17. Ta KN, Sabot F, Adam H, Vigouroux Y, De Mita S, Ghesquiere A, Do NV, Gantet P, Jouannic S. miR2118-triggered phased siRNAs are differentially expressed during the panicle development of wild and domesticated African rice species. Rice (N Y). 2016;9(1):10,

18. Xia R, Xu J, Arikit S, Meyers BC. Extensive families of miRNAs and PHAS loci in Norway spruce demonstrate the origins of complex phasiRNA networks in seed plants. Mol Biol Evol. 2015;32(11):2905-18.

19. Fan Y, Yang J, Mathioni SM, Yu J, Shen J, Yang X, Wang L, Zhang Q, Cai Z, Xu C, et al. PMS1T, producing phased small-interfering RNAs, regulates photoperiod-sensitive male sterility in rice. Proc Natl Acad Sci U S A. 2016: 113(52):15144-9.

20. Xia R, Zhu H, An YQ, Beers EP, Liu Z. Apple miRNAs and tasiRNAs with novel regulatory networks. Genome Biol. 2012;13(6):R47.

21. Lin JS, Lin CC, Lin HH, Chen YC, Jeng ST. MicroR828 regulates lignin and $\mathrm{H} 2 \mathrm{O} 2$ accumulation in sweet potato on wounding. The New Phytologist. 2012;196(2):427-40.

22. Qu D, Yan F, Meng R, Jiang X, Yang H, Gao Z, Dong Y, Yang Y, Zhao Z. Identification of MicroRNAs and their targets associated with fruit-bagging and subsequent sunlight re-exposure in the "granny smith" apple exocarp using high-throughput sequencing. Front Plant Sci. 2016;7:27.

23. Luo QJ, Mittal A, Jia F, Rock CD. An autoregulatory feedback loop involving PAP1 and TAS4 in response to sugars in Arabidopsis. Plant Mol Biol. 2012; 80(1):117-29.

24. Guan X, Pang M, Nah G, Shi X, Ye W, Stelly DM, Chen ZJ. miR828 and miR858 regulate homoeologous MYB2 gene functions in Arabidopsis trichome and cotton fibre development. Nature. Communications. 2014;5:3050.

25. Yoshikawa M, Peragine A, Park MY, Poethig RS. A pathway for the biogenesis of trans-acting siRNAs in Arabidopsis. Genes Dev. 2005;19(18): 2164-75.

26. Komiya R, Ohyanagi H, Niihama M, Watanabe T, Nakano M, Kurata N, Nonomura K. Rice germline-specific Argonaute MEL1 protein binds to phasiRNAs generated from more than 700 lincRNAs. Plant J. 2014;78(3): 385-97.

27. Xie F, Jones DC, Wang Q, Sun $R$, Zhang B. Small RNA sequencing identifies miRNA roles in ovule and fibre development. Plant Biotechnol J. 2015;13(3): 355-69.

28. $\mathrm{Hu} H, \mathrm{Yu}$ D, Liu H. Bioinformatics analysis of small RNAs in pima (Gossypium barbadense L.). PloS One. 2015;10(2):e0116826.

29. Wang S, Wang JW, Yu N, Li CH, Luo B, Gou JY, Wang LJ, Chen XY. Control of plant trichome development by a cotton fiber MYB gene. Plant Cell. 2004;16(9):2323-34.

30. Shangguan $X X, X u B, Y u Z X$, Wang $L J$, Chen XY. Promoter of a cotton fibre MYB gene functional in trichomes of Arabidopsis and glandular trichomes of tobacco. J Exp Bot. 2008;59(13):3533-42.

31. Howell MD, Fahlgren N, Chapman EJ, Cumbie JS, Sullivan CM, Givan SA, Kasschau KD, Carrington JC. Genome-wide analysis of the RNA-DEPENDENT RNA POLYMERASE6/DICER-LIKE4 pathway in Arabidopsis reveals dependency on miRNA- and tasiRNA-directed targeting. Plant Cell. 2007; 19(3):926-42.

32. Nonomura K, Morohoshi A, Nakano M, Eiguchi M, Miyao A, Hirochika H, Kurata N. A germ cell specific gene of the ARGONAUTE family is essential for the progression of premeiotic mitosis and meiosis during sporogenesis in rice. Plant Cell. 2007;19(8):2583-94.

33. Petsch K, Manzotti PS, Tam OH, Meeley R, Hammell M, Consonni G, Timmermans MC. Novel DICER-LIKE1 siRNAs bypass the requirement for DICER-LIKE4 in maize development. Plant Cell. 2015;27(8):2163-77.

34. Borges F, Martienssen RA. The expanding world of small RNAs in plants. Nat Rev Mol Cell Biol. 2015;16(12):727-41. 
35. Talmor-Neiman M, Stav R, Klipcan L, Buxdorf K, Baulcombe DC, Arazi T. Identification of trans-acting siRNAs in moss and an RNA-dependent RNA polymerase required for their biogenesis. Plant J. 2006;48(4):511-21.

36. Donaire L, Pedrola L, Rosa Rde L, Llave C. High-throughput sequencing of RNA silencing-associated small RNAs in olive (Olea europaea L.). PloS One. 2011;6(11):e27916.

37. Wan LC, Wang F, Guo X, Lu S, Qiu Z, Zhao Y, Zhang H, Lin J. Identification and characterization of small non-coding RNAs from Chinese fir by high throughput sequencing. BMC Plant Biol. 2012;12:146.

38. Xia R, Meyers BC, Liu Z, Beers EP, Ye S, Liu Z. MicroRNA superfamilies descended from miR390 and their roles in secondary small interfering RNA biogenesis in Eudicots. Plant Cell. 2013;25(5):1555-72.

39. Xue W, Wang Z, Du M, Liu Y, Liu JY. Genome-wide analysis of small RNAs reveals eight fiber elongation-related and 257 novel microRNAs in elongating cotton fiber cells. BMC Genomics. 2013:14:629.

40. Katiyar A, Smita S, Muthusamy SK, Chinnusamy V, Pandey DM, Bansal KC. Identification of novel drought-responsive microRNAs and trans-acting siRNAs from Sorghum bicolor (L.) Moench by high-throughput sequencing analysis. Frontiers in. Plant Sci. 2015;6:506.

41. Lin Y, Lin L, Lai R, Liu W, Chen Y, Zhang Z, XuHan X, Lai Z. MicroRNA390-directed TAS3 cleavage leads to the production of tasiRNA-ARF3/4 during somatic embryogenesis in Dimocarpus longan Lour. Front Plant Sci. 2015;6:1119.

42. Zhai J, Jeong D-H, De Paoli E, Park S, Rosen BD, Li Y, González AJ, Yan Z Kitto SL, Grusak MA. MicroRNAs as master regulators of the plant NB-LRR defense gene family via the production of phased, trans-acting siRNAs. Genes Dev. 2011;25(23):2540-53.

43. Arribas-Hernandez L, Marchais A, Poulsen C, Haase B, Hauptmann J, Benes V, Meister $\mathrm{G}$, Brodersen P. The slicer activity of ARGONAUTE1 is required specifically for the phasing, not production, of trans-acting short interfering RNAs in Arabidopsis. Plant Cell. 2016;28(7):1563-80.

44. Endo Y, Iwakawa HO, Tomari Y. Arabidopsis ARGONAUTE7 selects miR390 through multiple checkpoints during RISC assembly. EMBO Rep. 2013;14(7):652-8.

45. Guan X, Lee JJ, Pang M, Shi X, Stelly DM, Chen ZJ. Activation of Arabidopsis seed hair development by cotton fiber-related genes. PLoS One. 2011;6(7): e21301.

46. Wan Q, Guan X, Yang N, Wu H, Pan M, Liu B, Fang L, Yang S, Hu Y, Ye W, et al. Small interfering RNAs from bidirectional transcripts of GhMML3_A12 regulate cotton fiber development. The New Phytologist. 2016;210(4):1298310. https://doi.org/10.1111/nph.13860.

47. Wang X-J, Gaasterland T, Chua N-H. Genome-wide prediction and identification of cis-natural antisense transcripts in Arabidopsis thaliana. Genome Biol. 2005;6(4):R30.

48. Yang $X$, Wang L, Yuan D, Lindsey $K$, Zhang $X$. Small RNA and degradome sequencing reveal complex miRNA regulation during cotton somatic embryogenesis. J Exp Bot. 2013;64(6):1521-36. https://doi.org/ 10.1093/jxb/ert013.

49. Zhang T, Hu Y, Jiang W, Fang L, Guan X, Chen J, Zhang J, Saski CA, Scheffler BE, Stelly DM, et al. Sequencing of allotetraploid cotton (Gossypium hirsutum L. acc. TM-1) provides a resource for fiber improvement. Nat Biotechnol. 2015:33(5):531-7.

50. Livak KJ, Schmittgen TD. Analysis of relative gene expression data using real-time quantitative $P C R$ and the 2(-Delta Delta $C(T))$ method. Methods. 2001;25(4):402-8.

51. Kawahara Y. Quantification of adenosine-to-inosine editing of microRNAs using a conventional method. Nature Protocol. 2012;7(7):1426-37.

52. Langmead B, Trapnell C, Pop M, Salzberg SL. Ultrafast and memory-efficient alignment of short DNA sequences to the human genome. Genome Bbiology. 2009;10(3):R25.

\section{Publisher's Note}

Springer Nature remains neutral with regard to jurisdictional claims in published maps and institutional affiliations.

Ready to submit your research? Choose BMC and benefit from:

- fast, convenient online submission

- thorough peer review by experienced researchers in your field

- rapid publication on acceptance

- support for research data, including large and complex data types

- gold Open Access which fosters wider collaboration and increased citations

- maximum visibility for your research: over $100 \mathrm{M}$ website views per year

At $\mathrm{BMC}$, research is always in progress.

Learn more biomedcentral.com/submissions 